\section{Questión}

Periodismo / Comunicación ISSN 1669.6581

Un debate sobre trabajo no remunerado en la economía popular

Camila Stefanetti

Question/Cuestión, Nro.70, Vol.3, diciembre 2021

ISSN: 1669-6581

URL de la Revista: https://perio.unlp.edu.ar/ojs/index.php/question/

IICom -FPyCS -UNLP

DOI: https//doi.org/10.24215/16696581e641

\title{
Un debate sobre trabajo no remunerado en la economía popular
}

\section{A debate on unpaid work in the popular economy}

\section{Camila Stefanetti}

Universidad de Buenos Aires, Facultad de Ciencias Sociales Centro de Innovación de los Trabajadores (CITRA/UMET/CONICET)

Argentina

stefanetticamila@gmail.com http://orcid.org/0000-0001-7392-7315

\section{Resumen}

Partiendo de tres líneas teóricas: economía feminista, antropología política y perspectiva interseccional, el objetivo del artículo es brindar elementos para debatir sobre la visibilización del trabajo no remunerado en el sector de la economía popular. Para ello, se realiza una revisión bibliográfica sobre investigaciones que abordan trabajo no remunerado y organizaciones populares. A partir de las mismas se organizan dos ejes: aquellas que abordan la distribución desigual de las tareas productivas y reproductivas; y las que abordan la participación de las mujeres en la economía popular. 
Palabras claves: Trabajo no remunerado; Organizaciones sociales; Economía Popular; Revisión Bibliográfica

Abstract

Starting from three theoretical lines: feminist economics, political anthropology and intersectional perspective, the objective of the article is to provide elements to debate on the visibility of unpaid work in the popular economy sector. To do this, a bibliographic review is carried out on research that addresses unpaid work and popular organizations. From these two axes are organized: those that address the unequal distribution of productive and reproductive tasks; and those that address the participation of women in the popular economy.

Keywords: Unpaid work; Social organizations; Popular economy; Bibliographic review

\section{Introducción}

En el presente artículo se pretende brindar elementos para debatir sobre la visibilización del trabajo no remunerado en el sector de la economía popular, para ello se realiza una revisión bibliográfica sobre investigaciones académicas que aborden la temática del trabajo no remunerado en organizaciones sociales de la economía popular. El recorrido tanto de bibliografía reciente, como de las líneas teóricas más instaladas permitirá plantear a un futuro cercano una discusión más consolidada y sólida sobre las demandas en torno a la valorización y visibilización del trabajo no remunerado generadas dentro del sector de la economía popular.

A lo largo de este artículo se verá, en un primer apartado la contextualización y conceptualización de la problemática a abordar. Cómo a partir de los procesos políticos y sociales sucedidos en los años noventa y luego, con la llegada de gobiernos posneoliberales al país, se instalan nuevas lógicas de trabajo colectivas y autogestionadas. Asimismo, se analizará cómo fue el rol de las mujeres en las organizaciones sociales en ese contexto, hasta llegar al 2015 con el ciclo de movilización del Ni Una Menos. Allí, se observará como se establece nuevamente en agenda y con gran masividad, las demandas de género, observando específicamente las discusiones y demandas que presenta el feminismo popular en torno a la visibilización de las tareas de cuidado y trabajo no remunerado. 
En un segundo momento, estarán expuestas las diferentes líneas teóricas desde las cuales se toma posición para analizar la temática elegida, las mismas son: la economía feminista, la cual problematiza los límites entre lo productivo y lo reproductivo; la antropología política, que analiza las organizaciones sociales de la economía popular como categorías de la práctica; y por último la teoría interseccional, que analiza el entrecruzamiento de diversas desigualdades, en este caso entre género y clase.

En un tercer apartado, se expondrán varios estudios y artículos académicos organizados en dos ejes: en primer lugar, aquellas investigaciones que han abordado específicamente la problemática de la invisibilización de las tareas de cuidado y la distribución desigual de las tareas en base a la división sexual de trabajo predominante en la actualidad. Y, en segundo lugar, aquellas investigaciones que se han interrogado sobre las formas de participación de las mujeres en organizaciones sociales de la economía popular y sus prácticas cotidianas, problematizando el lugar que ocupan estas en los procesos de movilización social y modalidades de organización colectiva en la Argentina reciente. El rastreo bibliográfico se realizó a través del motor de búsqueda de Google Académico, Redalyc y Scielo, sumado a recomendaciones de pares.

Finalmente, se recuperarán los aspectos más salientes de los dos ejes construidos a partir de los antecedentes encontrados, poniendo en diálogo ambos conjuntos de estudios. Asimismo, en un último apartado, se abordarán algunas posibles áreas de vacancia.

\section{Conceptualización y contextualización sobre el trabajo no remunerado en la economía popular}

En las últimas décadas, las reconfiguraciones del capitalismo a escala global generaron un significativo proceso de heterogeneización de las formas de trabajo (Beck, 2000; Antunes, 2001; Castel, 2010) obligando a sectores crecientes de la población a desarrollar estrategias de supervivencia y generación de trabajo. En este contexto, los sectores populares en Argentina desarrollaron diversos procesos de organización a partir de la década de los noventa de diversas maneras, una de ellas fue en torno a la figura de trabajador desocupado, con una lógica de movimiento social anclado en el territorio, buscando una resolución colectiva de sus necesidades básicas, a partir de la movilización y de la autoorganización colectiva (Colectivo Situaciones, 2002a y 2002b; Svampa y Pereyra, 2003; Zibecchi, 2003; Delamata, 2004; Flores, 
2005; Retamozo, 2006; Merklen, 2010; Manzano, 2011; Natalucci, 2018); otras de las experiencias de movilización social sobresalientes han sido las empresas y fábricas recuperadas que dieron lugar a procesos de autogestión colectiva (Fernández Álvarez y Partenio, 2010).

En este contexto de movilización, el Estado implementó una serie de políticas sociales que se propusieron intervenir sobre el problema del desempleo y la exclusión a partir de la transferencia condicionada de ingresos monetarios, alguna de ellas son el Plan Trabajar I, II y III y el Plan Jefas y Jefes de Hogar Desocupados (PJJHD). En esas circunstancias, se sucede un nuevo ciclo político llamado "giro a la izquierda" (Levitsky y Roberts, 2011), "marea rosa" (Álvarez, et. al, 2017) o "posneoliberal" (Sader, 2009; Grugel y Riggiorizzi, 2012), allí el Estado impulsó una serie de programas sociales(1) como política de generación de empleo e "inclusión social" (Hopp, 2013), definidas en oposición a los programas "asistencialistas" y "focalizados" de las décadas previas (Hintze, 2007; Vuotto, 2008; Grassi, 2012).

A pesar de las políticas públicas creadas como respuestas al problema del desempleo, un porcentaje significativo de la clase trabajadora, lejos de ser reabsorbido como parte del mercado de trabajo por medio de un empleo asalariado, pasó a engrosar las filas del denominado sector "informal" de la economía, insertándose en circuitos de subcontratación, accediendo a empleos precarios o bien pasando a integrar cooperativas de trabajo impulsadas desde el Estado. En este marco, se crea en el 2011 la Confederación de Trabajadores de la Economía Popular (CTEP),(2) como confluencia de un proceso de organización que incluye, un conjunto heterogéneo de organizaciones sociales y políticas (Abal Medina, 2016; Fernández Álvarez, 2016; Natalucci, 2018), como así también a trabajadores y trabajadoras de empresas recuperadas, cooperativas de "cartoneros", vendedores ambulantes y ferias populares, cooperativas impulsadas tanto por organizaciones sociales como cooperativas conformadas en el marco de programas estatales.

En este punto, es preciso mencionar que varias autoras han destacado la presencia mayoritaria de mujeres en las organizaciones sociales de la economía popular (Partenio, 2005; Faur y Gherardi, 2005; Fernández Álvarez, 2006; Pautassi, 2009; Fernández Álvarez y Partenio, 2010; Zibecchi, 2013; Gago, 2019). Se observa como en un principio las mujeres de organizaciones sociales percibían planes sociales a cambio de ciertas prestaciones mayoritariamente asociadas con las responsabilidades del cuidado de los hijos y del trabajo no 
remunerado desarrollado en la esfera privada. A partir del 2013 y de la alta feminización del Programa de Ingreso Social con Trabajo, surge el Programa Ellas Hacen,(3) destinado a mujeres en situaciones de vulnerabilidad socio ocupacional. El desarrollo de esta línea de intervención específica destinada a mujeres se propuso el empoderamiento de las mismas, incorporando una perspectiva de género como eje transversal del programa.(4) La creación de este programa se fundamentó en la observación del impacto que la participación en cooperativas de trabajo tuvo para las mujeres, destacando aspectos como la ruptura de estereotipos laborales asociados al género (MDSN, 2015; Hopp, 2015) y el fomento de la independencia económica (Hintze, 2018). Pese a estos cambios en las políticas sociales y la presencia mayoritaria de las mujeres en las organizaciones sociales de la economía popular, se sostiene una división sexual del trabajo que les asigna tareas vinculadas al trabajo doméstico y reproductivo no remunerado como la cocina, comedores populares, huertas, cuidado de niños y tareas educativas (Zibecchi, 2013; Korol, 2016; Gago, 2019; Díaz Lozano, 2020), circunscribiéndose dentro del hogar o en los barrios, mientras que los varones ocupan lugares de decisión y representación política (Svampa y Pereyra, 2003), generando una segregación sexo/genérica, tanto horizontal como vertical (Tabbush y Caminiotti, 2015).

$\mathrm{Si}$ bien los procesos de movilización feminista tienen larga data en nuestro país (Molyneux, 2003; Barrancos, 2007; Tarducci y Rifkin, 2010; Di Marco, 2011), a mediados del 2015 se masifican con las demandas recuperadas por el Ni Una Menos, debido entre otras cosas al crecimiento de casos de femicidio (Sciortino, 2018a; Natalucci y Rey, 2018; Stefanetti, 2019). En un principio el eje de las manifestaciones fue el cese de femicidios, luego se expandieron a demandas más amplias, como el derecho al aborto seguro legal y gratuito, la aplicación de la Ley 26.485 de protección integral para prevenir, sancionar y erradicar la violencia contra las mujeres y a demandas vinculadas a la producción y reproducción de la vida como el techo y la escalera de cristal (Rodríguez, 2015; Sciortino, 2018a; Gago, 2019). En este contexto, las demandas por el reconocimiento del trabajo no remunerado fueron cobrando mayor centralidad en la agenda de las organizaciones de la economía popular, entendiendo que este recae sobre las mujeres principalmente de sectores populares, dejando en evidencia la intersección entre género y clase (Korol, 2016; Scocco, 2017). De esta manera, a inicios del 2020 la Unión de Trabajadores de la Economía Popular (UTEP, ex CTEP) a través de su Secretaría de Mujeres y Diversidad, exige demandas como: la Ley de emergencia por violencia 
de género, un subsidio para que las víctimas de violencia machista, el reconocimiento del trabajo de las promotoras en prevención de la violencia y promoción de derechos, una Red de Casas sin Violencia, entre otras. Asimismo, entre las demandas más vinculadas a cuidados y al sector sociocomunitario se impulsaron demandas como el reconocimiento salarial de las promotoras territoriales, la regularización inmediata y salarios dignos para todas las trabajadoras de casas particulares, una política integral de cuidados y asistencia profesional en los barrios populares. Con el advenimiento de la pandemia del COVID 19, se triplicó el trabajo de las mujeres en comedores y en tareas relacionadas a la salud, género, consumo problemático y apoyo escolar, al tiempo que comenzaron a brindar información sobre protocolos, testeos y vacunación.(5) En este contexto, una de las principales reivindicaciones del sector fue el reconocimiento de las trabajadoras sociocomunitarias como esenciales, reforzando la demanda por la remuneración del trabajo reproductivo a lo que se sumó la vacunación prioritaria. Así, la problematización de estas demandas y su proceso de construcción señala la sobrecarga de tareas, que al no estar reconocidas generan una mayor precariedad sobre sus vidas. La problematización de estas demandas deja expuesta la esencialidad del trabajo sociocomunitario, y la sobrecarga de tareas, que, al no estar reconocidas ni remuneradas, generan una mayor precariedad sobre sus vidas.

\section{Tres líneas teóricas que llevan a la visiblizanción de las demandas en torno al trabajo no remunerado: Antropología Política, Economía Feminista e interseccionalidad}

El problema de la visibilización del trabajo no remunerado y cómo se conforman sus respectivas demandas en la economía popular, puede abordarse desde ciertas líneas teóricas, una de ellas es la antropología política. Siguiendo la perspectiva adoptada por Manzano, Fernández Álvarez, Triguboff y Gregoric (2008), Grimberg (2009), Grimberg, Fernández Álvarez y Rosa (2009) y Fernández Álvarez (2014) se estudia a las organizaciones colectivas, como un ámbito para analizar más allá de construcciones hegemónicas, esto implica conceptualmente desplazar la mirada de la acción o el actor hacia el proceso y las relaciones en el que estas acciones cobran lugar.

Siguiendo esta perspectiva, se analiza el proceso de construcción de demandas y el lenguaje utilizado, como un modo específico de hacer política, confluyendo y tensionando 
tradiciones sociales y políticas, delimitadas por modalidades de intervención estatal (Fernández Álvarez, 2007). Aquí, el análisis de las interacciones entre prácticas estatales y modalidades de organización colectiva se realiza abordando el tejido de complementariedades, contradicciones e interdependencias que modelan tanto el sentido de las luchas como la orientación de las políticas (Grimberg, Fernández Álvarez y Carvalho Rosa, 2009; Fernández Álvarez, 2014). En esta dirección, Grimberg (2009) propone hablar de relaciones de hegemonía (en plural) ya que estas relaciones implican un proceso en el cual tanto dominantes como subalternos son activos, generando un proceso de mutuas apropiaciones y resignificaciones. Desde esta perspectiva, Fernández Álvarez (2015) busca contribuir a una conceptualización de las prácticas colectivas como un hacer juntos(as). Esta conceptualización da cuenta del carácter necesariamente contingente, contradictorio, fluido y parcial de estas prácticas sin por ello desconocer el modo en que ese hacer (juntos) requiere ser cristalizado en narrativas donde "lo colectivo" cobra sentido y presencia. Siguiendo esta línea, las prácticas de gestión colectiva, se reconstruyen capturando su carácter simultáneamente direccionado e indeterminado, proyectado y emergente (Fernández Álvarez, Gaztañaga y Quirós, 2017). En este sentido, se busca comprender a las organizaciones sociales de la economía popular como categorías de la práctica, capturando de manera vívida su carácter contradictorio, donde se desarrollan procesos y lenguajes, que, a la vez, internalizan formas de dominación y espacios de autonomía, como así también superponen relaciones de poder y prácticas igualitarias. Esta línea de trabajos antropológicos permitirá capturar etnográficamente aquello que se produce en los encuentros entre mujeres de organizaciones de la economía popular y agencias estatales y los modos en que estas interacciones se inscriben en trayectorias de vida y procesos organizativos más amplios

Un segundo conjunto de trabajos recuperados pertenece a la corriente de la economía feminista, aquí Pérez Orozco (2014) parte de una visión integral del trabajo, estudiando cómo se organiza y garantiza la reproducción de la vida colectiva e intentando reestructurar las relaciones sociales en pos de una menor desigualdad entre hombres y mujeres (Carrasco, 2012; Rodríguez Enríquez, 2015; Federici, 2016). La noción de división sexual del trabajo es una piedra angular para entender las demandas de estos sectores. Estas nuevas formas organizacionales trastocan las esferas de lo productivo y reproductivo, dándole centralidad a los conceptos de hogar y comunidad, exigiendo su visibilización y valoración. Federici (2016) 
plantea una revalorización del trabajo reproductivo para que sea asumido colectivamente en un marco de gestión no monopolizado por el Estado ni por el mercado, sino por las comunidades. Asimismo, Pérez Orosco (2014) trabaja de manera extensa la visibilización del ámbito reproductivo, mostrando que las mujeres que no están en el mercado laboral, bien lejos de estar inactivas, están muy presentes en la economía. Por su parte Nancy Fraser (2015) afirma que estos trabajos, afectivos y materiales, sin remuneración, son indispensables para la sociedad, sin ellos no habría cultura, ni economía ni organizaciones políticas.

Una tercera línea de investigación refiere a la intersección entre la economía popular y la economía feminista dando cuenta cómo a través de la participación de mujeres en procesos de organización colectiva, se redefinen los límites entre lo productivo y lo reproductivo. (Carrasco, 2012; Espinosa, 2013; Pacífico, 2017, Frega, 2019; Díaz Lozano, 2019; Sciortino, 2018b). Gago (2019) advierte una feminización de esas economías y de esos trabajos, frente a la presencia pública de las mujeres como un actor económico relevante, al mismo tiempo que se "feminizan" tareas desarrolladas en esa misma economía informal por los varones. La autora, identifica la afinidad histórica entre economía feminista y economía popular tiene que ver con la politización de la reproducción social desde la práctica política en el marco de la crisis. A partir de esta intersección es menester recuperar al feminismo popular, el cual según Claudia Korol (2016) en Argentina tiene sus inicios a partir de la segunda ola feminista durante la década del 1970 en Buenos Aires, los Encuentros Nacionales de Mujeres, los movimientos de desocupados que resistieron al neoliberalismo y el activismo en torno a los derechos humanos (Korol, 2016; Laudano, 2017; Gago, 2018; Sosa, Menéndez, Bascuas, 2018; Martínez Franzoni 2018). Este feminismo toma mayor visibilización a partir de la avanzada del capital y el neoliberalismo a comienzos del 2015 en la región, recuperando experiencias solidarias de sobrevivencia, de trabajo colectivo y creativo, sin patrones, e intentando no reproducir modelos de orden jerárquico y autoritario. En este sentido, aquellas organizaciones sociales que se definen como parte del feminismo popular han denunciado que las medidas económicas recientes tuvieron especial impacto en las vidas de mujeres, dificultando la capacidad de organización en las casas, en los barrios y en los territorios, poniendo en riesgo su autonomía económica. Aquí, es menester hacer mención al concepto de interseccionalidad acuñado por Crenshaw (2012), este analiza de manera compleja la forma en que ciertas categorías se imbrican y se relacionan con las estructuras de poder generando formas específicas de 
desigualdad, específicamente las demandas del sector mencionado como feminista popular presentan una interseccionalidad entre las categorías de género y clase que producen sexismo y clasismo (Elizalde, 2006; Valdivieso, 2014; Viveros, 2016).

A grandes rasgos, la formulación de la problemática a abordar - la valorización y visibilización del trabajo no remunerado dentro de las organizaciones sociales de la economía popular - pone en diálogo con dos conjuntos de estudios: en primer lugar, aquellas investigaciones que han abordado específicamente la problemática de la invisibilización de las tareas de cuidado y la distribución desigual de las tareas en base a la división sexual de trabajo predominante en la actualidad. $\mathrm{Y}$, en segundo lugar, aquellas investigaciones que se han interrogado sobre las formas de participación de mujeres en organizaciones sociales y sus prácticas cotidianas, problematizando el lugar que ocupan estas en los procesos de movilización social y modalidades de organización colectiva en la Argentina reciente.

\section{Tareas de cuidado y división sexual del trabajo}

El primer conjunto de estudio a abordar da cuenta de esta desigualdad en la distribución de tareas de cuidado no remunerativas, vinculándola a la división social del trabajo. Pautassi (2007) da cuenta que la división sexual del trabajo, como factor estructural, explica no solamente la asignación -casi exclusiva- de responsabilidades de cuidado a las mujeres en las familias, sino también la lógica de segregación en el interior del mercado que ubica a las mujeres en determinados sectores y ocupaciones. En este sentido, una serie de investigaciones han demostrado como los trabajos de cuidado permanecen asociados a lo femenino, asimismo dan cuenta como su invisibilización y escaso reconocimiento aún perduran (Arango Gaviria, 2011; Carrasco, 2011; Zibecchi, 2013).

Se observa una prolífica producción académica que ha puesto en el centro del análisis la cuestión de la provisión de cuidado en contextos de pobreza y desigualdad (Martínez Franzoni, 2008; Sojo, 2011; Esquivel, Faur y Jelin, 2012; Martínez Franzoni y Voorend, 2013; Marco Navarro y Rico, 2013). En ellas, se constata un excesivo condicionamiento que las responsabilidades de cuidado imponen a las mujeres pobres para su acceso y control de recursos económicos, donde se generan mayores responsabilidades de cuidado y, a su vez, 
poseen menores recursos para acceder a servicios mercantiles de cuidado. (Rodríguez Enríquez, 2012).

Esquivel, Faur y Jelin (2012) han señalado que la forma en que una sociedad encara la provisión de cuidados tiene implicancias significativas para el logro de la equidad de género, ya que las responsabilidades que surgen de dicha provisión suelen estar desigualmente distribuida entre varones y mujeres. Esta situación de desigualdad se encuentra socioeconómicamente estratificada y constituye un vector de reproducción de desigualdades (Rodríguez Enríquez, 2015). Acosta y Aguirre (2018), advierten en base a los datos arrojados por el INDEC, la situación de marginalidad en la que se encuentran las mujeres, la cual está marcada por múltiples desigualdades, en tanto están más desocupadas, más precarizadas, y más pobres sumado a que le dedican más tiempo en comparación a los hombres a las tareas domésticas no remuneradas, específicamente se observa que del total de personas que realizan tareas domésticas no remuneradas en Argentina, un $75 \%$ son mujeres y un $25 \%$ son varones.

Dentro de este eje, se observa la presencia de ciertos estudios que, desde un abordaje etnográfico, han abordado procesos de reproducción de la vida en sectores populares. De esta manera, Santillán (2010) estudia los procesos e iniciativas domésticas relativas a la socialización y la crianza de los niños y niñas y como en ese contexto se estrechan los diversos espacios que exceden el mundo "privado" de la vida doméstica. Zibecchi (2013) y Paura y Zibecchi (2014) analizan el funcionamiento de organizaciones sociales y comunitarias, su origen, sus prácticas y saberes, como así también la reconfiguración de espacios comunitario que atienden a una demanda específica de cuidado en la primera infancia y en contextos de pobreza. En este sentido, Pacífico (2017) indaga cómo se redefinen los límites entre lo productivo y lo reproductivo en el trabajo de las cooperativas. Sciortino (2018b) incorpora aportes de los estudios de género y feministas realizando un exhaustivo análisis sobre organizaciones colectivas en torno al trabajo entre mujeres de sectores populares y espacios comunitarios. El análisis de estas prácticas comunitarias, cooperativistas y mutualistas, en estos sectores, da cuenta de cómo la noción de casa toma relevancia y deja de ser un lugar pasivo (Pacífico, 2019).

\section{Participación de mujeres en organizaciones sociales de la economía popular}


Llegando al segundo eje, se observa cómo se han analizado arduamente las experiencias y prácticas de mujeres pertenecientes a organizaciones sociales a lo largo de estos últimos veinte años (Bidaseca, 2003; Causa, 2008; Di Marco, 2011; Monteagudo, 2011). Dentro de este eje se encuentran trabajos que han profundizado sobre el lugar que ocupan las temáticas y demandas de género dentro de las organizaciones sociales (Cross y Partenio, 2004; Partenio, 2008). Fernández Álvarez (2006) y Fernández Álvarez y Partenio (2010) se preguntan sobre la forma en que se actualizan, en el marco del proceso de recuperación de fábricas, los roles de género, como distribuyen tareas, horarios y responsabilidades; y analizan las implicancias que las redefiniciones entre estos espacios tuvieron para las mujeres.

Otro conjunto de investigaciones se ha propuesto indagar acerca de las subjetividades que se generan en tanto mujeres beneficiarias de programas sociales. (Molyneux, 2007; Lagos, 2008; Espinosa, 2013). Causa (2008) evidencia la adición de la militancia a las demás actividades productivas, reproductivas y de cuidado que realizan las mujeres, con la consiguiente "cuádruple carga de trabajo". Siguiendo esta línea, varios trabajos concuerdan en que en un contexto de militancia y, con una lógica que naturaliza la asociación entre mujer y cuidados, se incurre en una sobrecarga de trabajo para las "beneficiarias" (Pautassi, 2013; Zibecchi, 2013; Anzorena, 2013; Rodríguez Gusta, 2013; De Sena, 2014). Di Capua y Perbellini (2013), dan cuenta que muchas veces dentro de las cooperativas además de estar presente la estructura de dominación masculina propia de la sociedad patriarcal, se le suma otra estructura de dominación masculina sostenida sobre la autoridad del oficio, creando una doble inequidad en las relaciones sociales de género entre los miembros de un mismo colectivo de trabajo. Un conjunto de trabajos más recientes, han dado cuenta de cómo, a partir de la implementación de ciertas políticas públicas, se rompe con la idea de vincular a la mujer sólo a tareas domésticas dentro del hogar (Pacífico, 2017; Acosta y Aguirre, 2018; Gago, 2019). En esta línea, Pacífico (2019) aborda, a través de un enfoque etnográfico, las prácticas políticas colectivas desarrolladas por mujeres titulares del programa "Argentina Trabaja" considerando la relación entre las formas de organización puestas en marcha por las cooperativas y aquello que sucede en las viviendas. Al respecto, Hopp (2019), observa que a mayor participación laboral de las mujeres y el tiempo dedicado a las tareas establecidas por los programas de cooperativas, no 
se ven compensados del mismo modo por la participación de los varones en tareas del hogar y de cuidado, ni con la ampliación de servicios públicos que los provean.

\section{A modo de conclusión}

Para finalizar, es menester mencionar que este artículo buscó brindar elementos para profundizar y tener una base más sólida sobre la cual abordar la problemática de la valorización y visibilización de las tareas de cuidado y el trabajo no remunerado en las organizaciones sociales de la economía popular. Para ello, se realizó un breve esquema cronológico y contextual hasta llegar a la actualidad, en donde se reconstruyó los procesos políticos y sociales que dieron inicio a una nueva lógica de trabajo colectiva y a la formación de la CTEP y luego de la UTEP. Como se observó, desde el enfoque de la antropología política se conceptualizó a las prácticas colectivas como un hacer juntos(as). Asimismo, gracias a los aportes de la corriente teórica de la economía feminista se logró ampliar el panorama e incluir una visión más amplia que contemple a las tareas reproductivas y de cuidado. A partir de una mirada interseccional que entrecruza el género y la clase, se cuestionan estas prácticas dentro de los sectores populares, generando demandas en torno a las tareas de cuidado, dando cuenta así del feminismo popular.

Ahora bien, al analizar los trabajos académicos que se vinculan a la problemática abordada se llega a la conclusión de que existe dos ejes ordenadores: el primero, referido a las tareas de cuidado, su invisibilización y su desigual distribución según el sexo de la persona. En él, se puede destacar cómo esa distribución desigual del trabajo termina generando una lógica de segregación al interior del mercado laboral formal, en detrimento de las mujeres, debido al condicionamiento que las responsabilidades de cuidado generan en ellas. Siendo esta división socioeconómicamente estratificada, se observa que, en las mujeres de menores recursos, esta situación se agrava. Otro aspecto a destacar de este eje es como las tareas de cuidado, se encuentran en un límite muy difuso, donde se redefinen los límites entre lo productivo y lo reproductivo. El segundo eje, incorpora trabajos específicos que vinculan la participación de mujeres en organizaciones sociales. Allí se observó cómo las mujeres a pesar de estar dentro de una lógica colectiva de trabajo, siguen con una importante sobrecarga horaria al intentar distribuir su tiempo tanto en tareas productivas como reproductivas, como así también 
sumando la carga de la militancia. Asimismo, estar dentro de organizaciones sociales o cooperativas no garantiza que, a pesar de ser la cuestión de género un tema problematizado en esos espacios, se siga reproduciendo la lógica patriarcal y desigual de asociar a las mujeres a tareas reproductivas. Esta lógica viene desde sus mismos compañeros hombres o desde el Estado, reafirmando su rol vinculado a "cuidar" a través de políticas sociales. Aquí, es preciso mencionar que se han creado políticas como el Ellas Hacen, que intentan revertir esta mirada y darle más independencia, aunque a mi entender no lo han logrado de manera acabada.

Para finalizar, es preciso mencionar que a lo largo de la elaboración de este artículo se han percibido ciertas áreas de vacancia. Si bien hay trabajos que abordan desde una perspectiva espacial el problema de los límites entre lo productivo y reproductivo, no se han encontrado trabajos que avancen de una manera sustancial sobre la perspectiva temporal de este límite tan difuso. Esto quiere decir que, si bien hay trabajos que estudian la carga horaria que significan estas tareas para las mujeres, no se han encontrado trabajos que vinculen 0 analicen la sobreposición del tiempo dedicado a tareas productivas, reproductivas y de ocio. En este sentido, también se considera necesario realizar estudios que aborden de manera integral las demandas esgrimidas por las mujeres de organizaciones sociales de la economía popular en torno al trabajo no remunerado, cómo se construyen, su contenido y cómo inciden en ellas las subjetividades, trayectorias y prácticas cotidianas que transitaron y transitan las mujeres pertenecientes a organizaciones sociales de la economía popular.

\section{Referencias bibliográficas}

Acosta, A. y Aguirre, L. (2018). Economía feminista: teoría económica, desigualdad y políticas públicas. Un abordaje teórico y de caso. Ec-Revista de administración y economía. № 2, pp 5160.

Álvarez, S. et. al. (Ed.) (2017). Beyond civil society. Activism, Participation, and Protest in Latin America", Durham, UK: Duke University Press Durham.

Antunes, R. (2001). Adiós al trabajo? ensayo sobre las metamorfosis y la centralidad del mundo del trabajo. Cortez Editora. 
Anzorena, C. (2013). Mujeres en la trama del Estado: Una lectura feminista de las políticas públicas. Mendoza: Ediunc.

Arango Gaviria, L. (2011) El trabajo de cuidado: ¿servidumbre, profesión o ingeniería emocional? En Arango Gaviria, L. G. y Molinier, P. (eds.) El trabajo y la ética del cuidado. La carreta. Medellón. pp. 91-109.

Barrancos, D. (2007). Mujeres en la sociedad argentina: una historia de cinco siglos.Buenos Aires: Sudamericana.

Beck, U. (2000) Un nuevo mundo feliz. La precariedad del trabajo en la era de la globalización. Barcelona, Paidós.

Bidaseca, K. (2003). Piqueteras: identidad, política y resistencia". VII Jornadas de Historia de las Mujeres, II Congreso Iberoamericano de Estudios de Género. Salta.

Carrasco, C. (2012) Economía, trabajos y sostenibilidad de la vida. En Sostenibilidad de la vida. Aportaciones desde la Economía Solidaria, Feminista y Ecológica, de Yolanda Jubeto, Mertxe Larrañaga, \& Cristina Carrasco, 27- 42. Bilbao: REAS.

Carrasco, C. (2011) La economía del cuidado: planteamiento actual y desafíos pendientes. Revista de Economía Crítica. №11. pp. 205- 225.

Castel, R. (2010) El ascenso de las incertidumbres. Trabajo, protección, estatuto del individuo. Fondo de Cultura Económica.

Causa, A. (2008). Contexto. En Adriana Causa, y Julieta Ojam (comps.), Mujeres pi-queteras: Trayectorias, identidades, participación y redes, Buenos Aires, Ediciones Baobab, pp. 21-46.

Colectivo situaciones. (2002a) 19 y 20. Apuntes para el nuevo protagonismo social. Buenos Aires, De Mano en Mano. 
Colectivo situaciones. (2002b) La hipótesis 891. Más allá de los piquetes. Buenos Aires, De Mano en Mano.

Crenshaw, K. (2012). Interseccionalidad, políticas identitarias y violencia contra las mujeres de color. En Intersecciones: Cuerpos y sexualidades en la encrucijada. España. Editorial Bellaterra.

Cross, C. y Partenio, F. (2004). Mujeres y participación: Las organizaciones piqueteras y las relaciones de género. VI Jornadas de Sociología. Facultad de Ciencias Sociales, Universidad de Buenos Aires, Buenos Aires.

De Sena, A. (2014) Las mujeres ¿protagonistas de los programas sociales? Breves aportes a la discusión sobre la feminización de las políticas sociales. En De Sena, A. (ed.) Las políticas hechas cuerpo y lo social devenido emoción: lecturas sociológicas de las políticas sociales. Estudios Sociológicos Editora, Buenos Aires. pp. 99- 126.

Delamata, G. (2004). Los barrios desbordados, Libros del Rojas, Editorial de la Universidad de Buenos Aires. Buenos Aires: Eudeba.

Di Capua, M. y Perbellini, M. (2013). La división sexual y el sufrimiento en el trabajo: el caso de las empresas recuperadas. Trabajo y Sociedad. № 20, pp. 345-353. Disponible en: http://www.unse.edu.ar/trabajoysociedad

Di Marco, G. (2011). Las demandas en torno al aborto legal en Argentina y la constitución de nuevas identidades políticas. En G. Di Marco, \& C. Tabbush, Feminismos, democratización y democracia radical. Estudios de caso de América del Sur, Central, Medio Oriente y Norte de África. pp. 177-200. San Martin: UNSAM Edita. 
Díaz Lozano, J. (2020) Triple presencia femenina en torno a los trabajos: mujeres de sectores populares, participación política y sostenibilidad de la vida. Tempo e Argumento, Florianópolis. Vol 12, № 29.

Elizalde, S. (2006). "Mujeres que dan pelea. Dos experiencias de disputa política contra la represión y la exclusión en la Argentina contemporánea". Cuadernos de la Facultad de Humanidades y Ciencias Sociales: UNJU, № 31, pp 161-172.

Espinosa, C. (2013) Malentendidos productivos: 'Clivaje de género' y feminismo en una organización de trabajadores desocupados de Argentina". La ventana. Vol 4, № 37, pp 289323.

Esquivel, V; Faur, E. y Jelin, E. (2012). Hacia la conceptualización del cuidado: familia, mercado y estado. Las lógicas del cuidado infantil. Entre las familias, el Estado y el mercado. UNICEF- IDES. pp. 11- 44. Buenos Aires.

Faur, E. y Gherardi, N. (2005) El derecho al trabajo y la ocupación de las mujeres. Equipo Latinoamericano de Justicia y Género. Informe sobre Género y Derechos Humanos. Vigencia y respeto de los derechos de las mujeres en Argentina. Buenos Aires: Biblos.

Federici, S. (2016), Revolución en punto cero. Trabajo doméstico, reproducción y luchas feministas, Buenos Aires: Traficantes de sueños.

Fernández Álvarez, M. I. (2006) "Cuidar la fábrica, cuidar a los hijos. Roles de género, trabajo y acción colectiva a partir de un proceso de recuperación de fábricas de la Ciudad de Buenos Aires", Revista RUNA. № 26, pp. 7-26.

Fernández Álvarez, M. I. (2007). De la recuperación como acción a la recuperación como proceso: prácticas de movilización social y acciones estatales en torno a las recuperaciones de fábricas. Revista Cuadernos de Antropología Social. № 25, pp. 89-110. 
Fernández Álvarez, M. I. (2014). La política colectiva como problema antropológico: reflexiones desde el estudio de las cooperativas de trabajo como categorías de la práctica. QueHaceres. № 1, pp. 25-36.

Fernández Álvarez, M. I. (2015). Contribuciones antropológicas al estudio de las cooperativas de trabajo en la Argentina reciente. Revista del Centro de Estudios de Sociología del Trabajo (CESOT) №7, pp. 37-64.

Fernández Álvarez, M. I. (2016). Experiencias de precariedad, creación de derechos y producción colectiva de bienestar(es) desde la economía popular. Ensambles, № 4 y 5, pp.7289.

Fernández Álvarez, M. I., y Partenio, F. (2010). Empresas recuperadas en Argentina: producciones, espacios y tiempos de género. Tabula Rasa, №12, pp. 119-135.

Fernández Álvarez, M. I., GAZTAÑAGA , J.,QUIRÓZ, J. (2017). La política como proceso vivo: diálogos etnográficos y un experimento de encuentro conceptual. Revista Mexicana de Ciencias Políticas y Sociales, LXIII. № 231, pp.277-304.

Flores, T (comp.) (2005). De la culpa a la autogestión. Un recorrido del movimiento de trabajadores desocupados de la Matanza. Buenos Aires. Peña Lillo.

Fraser, N. (2015): Fortunas del Feminismo. Del capitalismo gestionado por el Estado a la crisis neoliberal. Madrid: traficantes de Sueños.

Frega, M. (2019). Mujeres y trabajos en el Conurbano reciente. Apuntes en clave feminista. Revista Latinoamericana de Antropología del Trabajo. Vol 3, № 5, pp.1-28.

Gago, V. (2018). \#Nosotras Paramos: notas hacia una teoría política de la huelga feminista. En V. Gago, R. Gutierrez Aguilar, S. Draper, M. Menendez Díaz, M. Montanelli, \& S. Rolnik. 8M Constelación Feminista. Buenos Aires: Tinta Limón. pp. 7-24. 
Gago, V. (2019). La potencia feminista: o el deseo de cambiarlo todo. Buenos Aires. Tinta Limón Ediciones.

Grassi, E. (2012) Política sociolaboral en la Argentina contemporánea. Alcances, novedades y salvedades. Ciencias Sociales. Vol. 1, № 135-136, pp. 185-198.

Grimberg, M, Fernández Álvarez, M. I. Rosa, M. (Eds) (2009). Estado y movilización social: estudios etnográficos en Argentina y Brasil. Buenos Aires: Co-edición Antropofagia.

Grimberg, M. (2009). Poder, políticas y vida cotidiana. Un estudio antropológico sobre protesta y resistencia social en el Área Metropolitana de Buenos Aires. Revista de Sociología e Política. № 32, pp.195-206.

Grugel J. y Riggiorizzi, P. (2012). Post-neoliberalism in Latin America: Rebuilding and Reclaiming the State after Crisis. Development and Change. Vol 43, № 1, pp. 1-21. Doi: 10.1111/j.1467-7660.2011.01746.x.

Hintze, S. (2007). Políticas sociales argentinas en el cambio de siglo. Conjeturas sobre lo posible. Buenos Aires: Espacio Editorial.

Hopp, M. (2013) ¿Cooperativas o planes sociales?: un análisis del proceso de implementación del Programa de Ingreso Social con Trabajo "Argentina Trabaja" en un municipio del Conurbano Bonaerense. Ponencia presentada en X Jornadas de Sociología de la UBA. Julio 2013.

Hopp, M. (2019). Políticas de promoción de cooperativas de trabajo en Argentina (Doctoral dissertation, Universidad de Buenos Aires).

Korol, C. (2016). Feminismos populares. Las brujas necesarias en los tiempos de cólera. Nueva Sociedad. № 265, pp. 142-152. 
Lagos, M. (2008). Vida cotidiana, ciudadanía y el género de la política. Cuadernos de Antropología Social. № 27, pp. 91-112.

Laudano, C. (2017). Movilizaciones \#Ni Una Menos y \#Vivas nos queremos en Argentina. Entre el Activismo digital y \#El feminismo lo hizo.13 Mundos de Mulheres \& Fazendo Gënero. № 11, pp. 1-12. Florianópolis.

Levitsky, S. y Roberts, K. (Eds.) (2011). The Resurgence of the Latin American Left. Baltimore, Estados Unidos: Johns Hopkins University Press.

Manzano, V. (2011). Cultura, política y movimiento estudiantil secundario en la Argentina de la segunda mitad del siglo XX. Propuesta educativa, (35), 41-52.

Manzano, V. (2013), La política en movimiento. Movilizaciones colectivas y políticas estatales en la vida del Gran Buenos Aires. Rosario: Prohistoria.

Manzano, V; Fernández Álvarez, M. I; Triguboff, M; Gregoric J.J. (2008) "Apuntes para la construcción de un enfoque antropológico sobre la protesta y los procesos de resistencia social" En Grimberg M, Fernandez, J., Fernández Álvarez M. I (comp,) Investiaciones en Antropología Social. Buenos Aires, coedición FFyL- Antropofagia pp. 41-62.

Marco Navarro, F. y Rico, N. (2013). Cuidado y Políticas Públicas: debates y estado de situación a nivel regional. En Laura Pautassi y Carla Zibecchi (comp.) Las fronteras del cuidado. Agenda, derechos e infraestructura. Buenos Aires: editorial Biblos - ELA.

Martínez Franzoni, J. (2008). ¿Arañando bienestar? Trabajo remunerado, protección social y familias en América Central. Buenos Aires: Clacso. 
Martínez Franzoni, J. y Voorend K. (2013). Desigualdades de género en los regímenes de bienestar latinoamericanos. En Laura Pautassi y Carla Zibecchi (comp.) Las fronteras del cuidado. Agenda, derechos e infraestructura. Buenos Aires: editorial Biblos - ELA.

Merkel, D. (2010). Pobres Ciudadanos. Buenos Aires. Gorla.

Molyneux, M. (2003). Movimientos de mujeres en América Latina. Estudio teórico comparado. Ediciones Cátedra: Madrid.

Molyneux, M. (2007) Change and continuity in Social Protection in Latin America Mothers at the Service of the State?" Gender and Development Programme Paper 1.

Monteagudo, G. (2011). Politics by Other Means: Rhizomes of Power in Argentina's Social Movements, Dissertations. Paper 420. University of Massachusetts-Amherst. [en línea], dis-ponible en <http://scholarworks.umass.edu/open_access_dissertations/420>, consultado el 12 de diciembre de 2014.

Natalucci, A. (2018). El neoliberalismo en acto: políticas sociales y experiencias organizativas en Argentina (2009-2016). Polis. Revista Latinoamericana. №49, pp. 1-19.

Natalucci, A. y Rey, J. (2018). ¿Una nueva oleada feminista? Agendas de género, repertorios de acción y colectivos de mujeres (Argentina, 2015-2018). Revista de estudios políticos y estratégicos, Vol 6, № 2, pp. 14-34.

Pacífico, F. (2017). Entre lo productivo y lo reproductivo. Un análisis etnográfico del trabajo de mujeres en cooperativas y programas sociales. Fazendo Género. №11, pp.1-13.

Pacífico, F. (2019). Casas, programas sociales y prácticas políticas colectivas Etnografía de experiencias cotidianas de mujeres titulares del "Argentina Trabaja". RUNA, archivo para las ciencias del hombre. Vol. 40, № 2, pp. 273-292. 
Partenio, F. (2008) «Una comparación entre los procesos de recuperación de fábricas en Argentina, Brasil y Uruguay» (mimeo).

Paura, V. y Zibecchi, C. (2014). Mujeres, ámbito comunitario y cuidado: Consideraciones para el estudio de relaciones en transformación. La aljaba versión On-line. №. 18, pp. 125-147.

Pautassi, L. (2009) Programas de transferencias condicionadas de ingresos ¿Quién pensó en el cuidado? La experiencia en Argentina. CEPAL, Santiago de Chile.

Pautassi, L. (2007). El cuidado como cuestión social desde un enfoque de derechos. Serie Mujer y Desarrollo. № 87. Santiago de Chile: Comisión Económica para América Latina y el Caribe (CEPAL). Recuperado de http://www.cepal.org/mujer/noticias/noticias/4/32494/serie87.pdf

Pautassi, L. (2013). El trabajo de cuidar y el derecho al cuidado. ¿Círculos concéntricos de la política social?. Revista Cátedra Paralela. №10, pp. 65-92. Rosario, Universidad Nacional de Rosario.

Pérez Orozco, A. (2014). Subversión feminista de la economía. Aportes para un debate sobre el conflicto capital-vida. Madrid: Traficantes de sueños.

Quirós, J. (2011). El porqué de los que van. Peronistas y piqueteros en el Gran Buenos Aires (una antropología de la política vivida). Buenos Aires: Antropofagia

Retamozo, M. (2006). El movimiento de los trabajadores desocupados en Argentina: cambios estructurales, subjetividad y acción colectiva en el orden social neoliberal. Argumentos: Estudios Críticos de la Sociedad. Vol. 19, № 50, pp. 145-166. Recuperado de http://www.scielo.org.mx/scielo.php?script=sci_arttext\&pid=S0187-57952006000100007 
Rodríguez Enríquez, C. (2012). La cuestión del cuidado: ¿El eslabón perdido del análisis económico? CEPAL, № 106 (LC/G. 2518-P), Santiago de Chile, Comisión Económica para América Latina y el Caribe (CEPAL). Publicación de las Naciones Unidas.

Rodríguez Enríquez, C. (2015), Economía feminista y economía del cuidado, Nueva Sociedad. № 256, pp. 30-44. Disponible en: http://nuso.org/media/articles/downloads/4102_1.pdf

Rodríguez, P. (2015). \#NiUnaMenos. Ciudad Autónoma de Buenos Aires: Planeta.

Rodríguez Gusta, A. (2013). ¿Destinatarias emprendedoras o beneficiarias dependientes?: Segmentaciones discursivas en la implementación municipal de una política social en la Argentina. Cuadernos de antropología social. № 37, pp. 139-169.

Sader, E. (2009). El nuevo topo. Los caminos de la izquierda latinoamericana. Buenos Aires, Argentina: Siglo XXI Editores.

Santillán, L. (2010). Las configuraciones sociales de la crianza en barrios populares del Gran Buenos Aires. Revista Latinoamericana de Ciencias Sociales, Niñez y Juventud. Vol.8, № 2, pp. 921-932.

Sciortino, S. (2018a). Consideraciones sobre el movimiento amplio de mujeres a partir del 'Ni una menos': Continuidad histórica, diversidad y trayectorias locales. Publicar. Vol. 16, № 24, pp. 27-47.

Sciortino, M. (2018b). Una etnografía sobre arreglos familiares, leonas y mujeres superpoderosas. Cuadernos de antropología social. №48, pp. 55-71.

Scocco, N. (2017). "La salud en los sectores populares". En AA.VV, Economía Popular. Los desafíos del trabajo sin patrón. Bs. As.: Colihue. 
Sojo, A. (2011). De la evanescencia a la mira: el cuidado como eje de políticas y de actores en América Latina. Serie seminarios y conferencias. № 67. Santiago de Chile: CEPAL.

Sosa, M., Menéndez, M., y Bascuas, M. (2018). "Experiencias de feminismo popular en el Cono Sur: reproducción de la vida y relaciones entre mujeres". En H. Mena Méndez, Las disputas por lo público en América Latina y el Caribe. Buenos Aires: Clacso.

Stefanetti, C. (2019). El ciclo de movilización \#NiUnaMenos: La recreación del movimiento de mujeres en Argentina (2015-2019) XIV Congreso Nacional de Ciencia Política. SAAP. Universidad Nacional de San Martín, Gral. San Martín.

Svampa, M. y Pereyra S. (2003) Entre la ruta y el barrio. Buenos Aires. Biblos.

Tabbush, C., y Caminiotti, M. (2015). Igualdad de género y movimientos sociales en la Argentina posneoliberal: la Organización Barrial Tupac Amaru. Perfiles latinoamericanos. Vol, 23, № 46, pp.147-171.

Tarducci, M. y Rifkin, D. (2010). Fragmentos de historia del feminismo en Argentina. En Chaher y Santoro (comp.). Las palabras tienen sexo II. pp. 17-39. Buenos Aires: Artemisa Comunicación

Valdivieso, M. (2014) "Otros tiempos y otros feminismos en América Latina y el Caribe. Alternativas desde el feminismo". En Feminismos para un cambio civilizatorio. CLACSO, Venezuela.

Viveros, V. (2016) La interseccionalidad: una aproximación situada a la dominación. Revista Debate Feminista, Vol. 52, pp. 1-17.

Vuotto, M. (2008) El alcance de las estrategias gubernamentales de promoción de la economía social en la Argentina. 27 Congreso Internacional del CIRIEC disponible en internet: http://conference.se-es.ca/wp-content/uploads/2008/02/mirta-vuotto-re.pdf 
Zibecchi, C. (2013) Trayectorias Asistidas Un abordaje de los programas sociales en Argentina desde el enfoque de género. Eudeba.

\section{Notas}

(1)Uno de ellos fue el Programa de Ingreso Social con Trabajo (PRIST) en el 2009 por medio del decreto № 1067 . Este promovía el desarrollo de capital humano y capacidades sociales de personas en situación de alta vulnerabilidad socioeconómica a través de la generación de oportunidades socioocupacionales" (MDS 2014, p. 6).

(2) La CTEP es creada como una herramienta gremial para aquellos trabajadores popularmente definidos como "informales", precarios", "subempleados" o "de subsistencia" quienes habiendo quedado fuera del mercado de empleo "se inventaron el trabajo para sobrevivir" (Fernández Álvarez, 2016)

(3) El Programa Ellas Hacen implementado desde el Ministerio de Desarrollo Social (MDS) está destinado a mujeres desocupadas, jefas de hogar monoparentales, que perciban la Asignación Universal por Hijo, preferentemente con tres o más hijos menores de 18 años o discapacitados, o que sufran violencia de género, vivan en barrios vulnerables y estén dispuestas a realizar, en primer término, actividades de capacitación y terminalidad educativa.

(4) Ministerio de Desarrollo Social (Resolución № 2055/2016).

(5) Según la última actualización del Registro Nacional de Trabajadoras y Trabajadoras de la Economía Popular (ReNaTEP), en mayo de 2021, las trabajadoras socio-comunitarias representan un $32 \%$.

(6) Las actividades llamadas esenciales son las consideradas de vital importancia para el funcionamiento del país durante la pandemia del COVID 19.

(7) Datos de la Encuesta Permanente de Hogares. 3er trimestre de 2018. 